\title{
LINEAR FUNCTIONAL DIFFERENTIAL EQUATIONS \\ POSSESSING SOLUTIONS WITH A GIVEN GROWTH RATE
}

\author{
RAVI P. AGARWAL AND ANDREI RONTÓ
}

Received 7 April 2004

We establish optimal, in a sense, conditions under which, for arbitrary forcing terms from a suitable class, a linear inhomogeneous functional differential equation in a preordered Banach space possesses solutions satisfying a certain growth restriction.

\section{Introduction}

When studying mathematical models of various dynamic phenomena, it is often desirable not only to prove the existence of a solution satisfying the given initial or boundary conditions but also to ensure that the solution in question possesses certain qualitative properties (e.g., has at least a prescribed number of zeroes on the given interval). It should be noted that, in most cases, the techniques commonly used to describe the qualitative behaviour of solutions do not provide any algorithm to find the solution itself.

In this paper, we prove two theorems which provide conditions under which a linear functional differential equation has a unique absolutely continuous solution passing through a given point, having a given growth rate, representable as a uniformly convergent functional series with the coefficients defined recursively, and positive in a certain sense. More precisely, we are interested in the solutions of the abstract functional differential equation

$$
u^{\prime}(t)=(\ell u)(t)+f(t), \quad t \in[a, b]
$$

satisfying the condition

$$
\sup _{t \in[a, b]} \frac{\|u(t)-c\|}{\phi(t)}<+\infty
$$

where $\phi:[a, b] \rightarrow \mathbb{R}$ is a given nonnegative continuous function possessing at least one zero on the interval $[a, b]$. Throughout the paper, we exclude from consideration the trivial case where $\phi(t)=0$ for all $t \in[a, b]$ and do not mention this explicitly in the statements. 
Here, $\ell: C([a, b], X) \rightarrow L([a, b], X)$ is a continuous linear operator, $X$ is a Banach space, $c$ is a given vector from $X$, and $f:[a, b] \rightarrow X$ is an arbitrary Bochner integrable function satisfying a certain growth restriction. By a solution of problem (1.1), (1.2), we mean an absolutely continuous abstract function $u:[a, b] \rightarrow X$ possessing property (1.2) and satisfying (1.1) almost everywhere on $[a, b]$.

The main purpose of this paper is to give optimal, in a sense, conditions under which problem (1.1), (1.2) is solvable for a sufficiently wide class of pairs $(c, f)$ from $X \times$ $L([a, b], X)$, and to provide a series expansion of a solution.

\section{Notation}

The following notation is used in the sequel.

(1) $\mathbb{R}=(-\infty,+\infty), \mathbb{N}=\{1,2, \ldots\}$.

(2) $\langle X,\|\cdot\|\rangle$ is a Banach space.

(3) $C([a, b], X)$ is the Banach space of continuous functions $u:[a, b] \rightarrow X$ endowed with the norm

$$
C([a, b], X) \ni u \longmapsto \max _{t \in[a, b]}\|u(t)\| .
$$

(4) $B([a, b], X)$ is the Banach space of bounded functions $u:[a, b] \rightarrow X$ endowed with the norm

$$
B([a, b], X) \ni u \longmapsto \sup _{t \in[a, b]}\|u(t)\| .
$$

(5) $C_{\phi}([a, b], X)$ is the Banach space of continuous functions $u:[a, b] \rightarrow X$ satisfying the condition

$$
\sup _{t \in[a, b]} \frac{\|u(t)\|}{\phi(t)}<+\infty .
$$

The norm in $C_{\phi}([a, b], X)$ is denoted by the symbol $\|\cdot\|_{\phi}$ and defined by the relation

$$
u \longmapsto\|u\|_{\phi}:=\sup _{t \in[a, b]} \frac{1}{\phi(t)}\|u(t)\| .
$$

(6) $L([a, b], X)$ is the Banach space of Bochner integrable functions $u:[a, b] \rightarrow X$ endowed by the norm

$$
L([a, b], X) \ni u \longmapsto \int_{a}^{b}\|u(t)\| d t .
$$

(7) $\mathscr{L}(X)$ is the algebra of all the bounded linear operators in $X$.

(8) $r(A)$ is the spectral radius of a linear operator $A$.

(9) $\geqslant_{K}$ and $\gg_{K}$ : see Definitions 3.3 and 3.5.

(10) blade K: see Definition 3.2 and formula (3.1).

(11) $\mathscr{P}_{K, \phi}(\tau, \Omega ;[a, b], X)$ : see Definition 3.9.

(12) $C_{K, \Omega, \phi}([a, b], X)$ : see formula (5.23). 


\section{Definitions and assumptions}

3.1. Wedges and cones. In this subsection, for reader's convenience, we briefly recall several basic definitions from the theory of linear semigroups in Banach spaces (see, e.g., $[3,5])$.

Definition 3.1. A nonempty closed set $K$ in a Banach space $X$ is called a linear semigroup [5], or a wedge [3], if the following conditions are satisfied:

(1) $K+K \subset K$;

(2) $\alpha K \subset K$ for an arbitrary $\alpha \in[0, \infty)$.

Here, by definition, we set $K+K:=\left\{x_{1}+x_{2} \mid\left\{x_{1}, x_{2}\right\} \subset K\right\}$ and, similarly, $\alpha K:=\{\alpha x \mid$ $x \in K\}$.

Definition 3.2. The set $K \cap(-K)$ is referred to as the blade [3] of the wedge $K$.

We will use the following notation for the blade:

$$
K \cap(-K)=\text { : blade } K \text {. }
$$

The presence of a wedge in a Banach space $X$ allows one to introduce a natural preordering there. More precisely, we introduce the following standard.

Definition 3.3. Two elements $\left\{x_{1}, x_{2}\right\} \subset X$ are said to be in relation $x_{1} \leqslant_{K} x_{2}$ if and only if they satisfy the relation $x_{2}-x_{1} \in K$.

In a similar way, the relation $\geqslant_{K}$ is introduced: $x_{1} \geqslant_{K} x_{2}$ if and only if $x_{2} \leqslant_{K} x_{1}$. Thus, we have $K=\left\{x \in X \mid x \geqslant_{K} 0\right\}$ and

$$
\text { blade } K=\left\{x \in X \mid 0 \leqslant_{K} x \leqslant_{K} 0\right\} .
$$

Definition 3.4. A wedge $K \subset X$ will be called proper if it does not coincide with the entire space $X$ and is different from the zero-dimensional subspace $\{0\}$.

Definition 3.5. A wedge $K \subset X$ is said to be solid [5] if its interior is nonempty.

In the case of a solid wedge $K$, following [5], we write $x \gg_{K} 0$ if and only if $x$ belongs to the interior of $K$.

Definition 3.6. The wedge $K$ is called a cone $[3,5]$ if blade $K=\{0\}$.

3.2. The set $\mathscr{B}_{K, \phi}(\tau, \Omega ;[a, b], X)$. Let $\tau$ be a point in $[a, b], \Omega$ a subset of $[a, b], K \subset X$ a wedge, and $\phi:[a, b] \rightarrow[0,+\infty)$ a continuous function. In what follows, we always assume that $K$ is a proper wedge.

Definition 3.7. An operator $\ell: C([a, b], X) \rightarrow L([a, b], X)$ is said to be $(\tau, \Omega, \phi)$-positive with respect to the wedge $K \subset X$ if

$$
\int_{\tau}^{t}(\ell u)(s) d s \geqslant_{K} 0 \quad \forall t \in[a, b] \backslash \Omega
$$


whenever the function $u$ from $C([a, b], X)$ satisfies the condition $(2.3)$ and is such that

$$
u(t) \geqslant_{K} 0 \quad \forall t \in[a, b] \backslash \Omega
$$

Recall that the sign $\geqslant_{K}$ is introduced by Definition 3.3.

Definition 3.8. A linear operator $\ell: C([a, b], X) \rightarrow L([a, b], X)$ will be called $\phi$-regular if there exists a nonnegative Lebesgue integrable function $\omega:[a, b] \rightarrow \mathbb{R}$ such that, for an arbitrary $u$ from $C_{\phi}([a, b], X)$, the following condition is satisfied:

$$
\|(\ell u)(t)\| \leq \omega(t) \sup _{s \in[a, b]} \frac{\|u(s)\|}{\phi(s)} \quad \forall t \in[a, b] .
$$

Note that all the linear operators determining the functional differential equations (1.1) arising in concrete applications satisfy the condition described by Definition 3.8 with a certain continuous function $\phi:[a, b] \rightarrow[0,+\infty)$.

Definition 3.9. By the symbol $\mathscr{B}_{K, \phi}(\tau, \Omega ;[a, b], X)$, where $\phi:[a, b] \rightarrow \mathbb{R}$ is a nonnegative continuous function, we denote the set of all continuous linear operators $\ell: C([a, b], X) \rightarrow$ $L([a, b], X)$ that are $\phi$-regular, $(\tau, \Omega, \phi)$-positive with respect to the wedge $K$ and, moreover, satisfy Assumptions 3.10 and 3.11 .

3.3. Two assumptions. We introduce the following two conditions imposed on the linear operator $\ell: C([a, b], X) \rightarrow L([a, b], X)$ in $(1.1)$.

Assumption 3.10. For an arbitrary continuous function $u:[a, b] \rightarrow X$, the inclusion $u([a, b] \backslash \Omega) \subset$ blade $K$ and condition (2.3) imply that

$$
\int_{\tau}^{t}(\ell u)(s) d s=0 \quad \forall t \in[a, b]
$$

Here and below, we use the notation $u(M):=\{u(t) \mid t \in M\}$ for $M \subset[a, b]$.

Assumption 3.11. For an arbitrary continuous function $u:[a, b] \rightarrow X$ satisfying condition (2.3), there exists a nonnegative constant $\beta_{u}$ such that

$$
\left\|\int_{\tau}^{t}(\ell u)(s) d s\right\| \leq \beta_{u} \phi(t) \quad \forall t \in[a, b] .
$$

Example 3.12. Let the operator $\ell: C([a, b], X) \rightarrow L([a, b], X)$ be given by the relation

$$
(\ell u)(t)=P(t) u(\omega(t)), \quad t \in[a, b]
$$

where $P:[a, b] \rightarrow \mathscr{L}(X)$ is integrable and $\omega:[a, b] \rightarrow[a, b]$ is measurable. Let $\Omega$ be a subset of $[a, b]$. Then Assumption 3.10 is satisfied provided that

$$
\begin{gathered}
\omega([a, b]) \subset[a, b] \backslash \Omega, \\
\operatorname{ker} P(t) \supset \text { blade } K \quad \forall t \notin \Omega .
\end{gathered}
$$


On the other hand, Assumption 3.11 holds whenever

$$
\operatorname{sign}(t-\tau) \int_{\tau}^{t}\|P(\xi)\| \phi(\omega(\xi)) d \xi \leq B \phi(t), \quad t \in[a, b]
$$

with some $B \in[0,+\infty)$.

\section{Conditions sufficient for the solvability of problem (1.1), (1.2)}

We consider problem (1.1), (1.2) in a Banach space $X$, in which we fix a certain proper wedge $K$. Throughout this section, $\phi:[a, b] \rightarrow[0,+\infty)$ is a given continuous function not identically equal to zero and such that $\phi^{-1}(0) \neq \varnothing$.

Theorem 4.1. Assume that the wedge $K$ is solid and the linear operator $\ell: C([a, b], X) \rightarrow$ $L([a, b], X)$ belongs to $\mathscr{B}_{K, \phi}(\tau, \Omega ;[a, b], X)$ for some set $\Omega \subset[a, b]$ such that $[a, b] \backslash \Omega$ is closed. Also suppose that there exists a continuous abstract function $y:[a, b] \rightarrow X$ satisfying the conditions

$$
\begin{gathered}
y(t) \gg_{K} 0 \quad \forall t \in[a, b] \backslash \Omega, \\
\sup _{t \in[a, b]} \frac{\|y(t)\|}{\phi(t)}<+\infty
\end{gathered}
$$

and, moreover, such that

$$
\int_{\tau}^{t}(\ell y)(s) d s \leqslant_{K} \alpha y(t) \quad \text { for every } t \in[a, b] \backslash \Omega
$$

with a certain constant $\alpha \in[0,1)$ and some $\tau \in \phi^{-1}(0)$.

Then, for arbitrary $c \in X$ and $f \in L([a, b], X)$ satisfying the condition

$$
\sup _{t \in[a, b]} \frac{1}{\phi(t)}\left\|\int_{\tau}^{t}[f(s)+(\ell c)(s)] d s\right\|<+\infty
$$

problem (1.1), (1.2) possesses a solution $u(\cdot)$ representable in the form of the uniformly convergent functional series

$$
u(t)=f_{\tau, c}(t)+\int_{\tau}^{t}\left(\ell f_{\tau, c}\right)(s) d s+\int_{\tau}^{t} \ell\left(\int_{\tau}^{\cdot}\left(\ell f_{\tau, c}\right)(\xi) d \xi\right)(s) d s+\cdots, \quad t \in[a, b],
$$

where

$$
f_{\tau, c}(t):=c+\int_{\tau}^{t} f(s) d s, \quad t \in[a, b]
$$

This solution, moreover, satisfies the initial condition

$$
u(\tau)=c .
$$


If, furthermore, the function $f \in L([a, b], X)$ and vector $c \in X$ are such that

$$
\int_{\tau}^{t} f(s) d s \geqslant_{K}-c \quad \forall t \in[a, b] \backslash \Omega,
$$

then solution (4.5) of problem (1.1), (1.2) also satisfies condition (3.4).

The expression $\ell c$ in condition (4.4) is understood as the value of $\ell$ on the constant function $[a, b] \rightarrow X$ identically equal to $c$.

Remark 4.2. Under the assumptions of Theorem 4.1, to every fixed $\tau$ involved in the conditions, exactly one solution of problem (1.1), (1.2) corresponds; that is, the problem mentioned cannot have two different solutions taking the same value at the point $\tau$.

Remark 4.3. It follows immediately from property (1.2) that a solution of problem (1.1), (1.2) has at least as many zeroes as the function $\phi$ does.

Remark 4.4. Condition (4.8) is satisfied, in particular, if $c \geqslant_{K} 0$ and the integrable function $f:[a, b] \rightarrow X$ satisfies the relation

$$
f(t) \operatorname{sign}(t-\tau) \geqslant_{K} 0 \quad \text { for a.e. } t \in[a, b] .
$$

In the case where the wedge $K$ is a cone, Theorem 4.1 yields the following statement.

Theorem 4.5. Assume that, for some solid cone $K$ in $X$ and a subset $\Omega$ of $[a, b]$ such that $[a, b] \backslash \Omega$ is closed, the linear operator $\ell: C([a, b], X) \rightarrow L([a, b], X)$ is $\phi$-regular, $(\tau, \Omega, \phi)$ positive with respect to $K$, satisfies Assumption 3.11, and, moreover, for an arbitrary function $u$ from $C([a, b], X)$ satisfying condition (2.3) and vanishing on the set $[a, b] \backslash \Omega$, condition (3.6) holds.

Then the existence of a continuous abstract function $y:[a, b] \rightarrow X$ satisfying conditions (4.1), (4.7), and (4.3) for some $\alpha \in[0,1)$ and $\tau \in \phi^{-1}(0)$ ensures that, for arbitrary $(c, f) \in$ $X \times L([a, b], X)$ satisfying condition (4.4), problem (1.1), (1.2) has a solution possessing property (4.7) and representable in the form (4.5), where $f_{\tau, c}$ is the function given by formula (4.6).

If, in addition, relation (4.8) holds for the function $f \in L([a, b], X)$ and vector $c \in X$, then solution (4.5) of problem (1.1), (1.2) also satisfies condition (3.4).

Remark 4.6. It follows from [1, Theorem 1] that, in the case where the cone $K$ is also normal [5] (i.e., there exists a constant $\delta \in(0,+\infty)$ such that $\left\|x_{1}+x_{1}\right\| \geq \delta$ whenever $\left\{x_{1}, x_{2}\right\} \subset K$ and $\left.\left\|x_{1}\right\|=\left\|x_{2}\right\|=1\right)$, the assumption on the continuity of $\ell$ in Theorem 4.5 is satisfied automatically.

Corollary 4.7. Suppose that $K$ is a solid cone in $X$, and the $\phi$-regular linear operator $\ell: C([a, b], X) \rightarrow L([a, b], X)$ is such that Assumption 3.11 is true and, moreover,

$$
(\ell u)(t) \operatorname{sign}(t-\tau) \geqslant_{K} 0 \quad \text { for a.e. } t \in[a, b]
$$

whenever the function $u$ from $C([a, b], X)$ satisfies relation (2.3) and the condition

$$
u(t) \geqslant_{K} 0 \quad \forall t \in[a, b] .
$$


Furthermore, assume that there exist a constant $\alpha \in[0,1)$ and a continuous abstract function $y:[a, b] \rightarrow X$ satisfying condition (4.2), the relation

$$
y(t) \gg_{K} 0 \quad \forall t \in[a, b]
$$

and the integral inequality

$$
\alpha y(t) \geqslant_{K} \int_{\tau}^{t}(\ell y)(s) d s \quad \text { for every } t \in[a, b]
$$

with some $\tau \in \phi^{-1}(0)$.

Then, for arbitrary $f \in L([a, b], X)$ and $c \in X$ satisfying condition (4.4), problem (1.1), (1.2) admits solution (4.5) with $f_{\tau, c}$ given by (4.6). The solution mentioned satisfies the initial condition (4.7). If, furthermore,

$$
\int_{\tau}^{t} f(s) d s \geqslant_{K}-c \quad \forall t \in[a, b]
$$

then the solution given by (4.5) also possesses property (4.11).

Remark 4.8. Conditions (4.3) and (4.13) in the statements formulated above are optimal in the sense that the inequality $\alpha<1$ for the constant $\alpha$ involved therein, generally speaking, cannot be replaced by the corresponding nonstrict inequality $\alpha \leq 1$.

Indeed, we consider the simplest scalar functional differential equation

$$
u^{\prime}(t)=u(1)+f(t), \quad t \in[0,1]
$$

together with the additional condition

$$
\sup _{t \in[0,1]} \frac{|u(t)-1|}{\sqrt{t}}<+\infty .
$$

Problem (4.15), (4.16), obviously, can be rewritten as (1.1), (1.2) with $X=\mathbb{R}, a=0$, $b=1, \phi(t):=\sqrt{t}, t \in[0,1]$, and the operator $\ell: C([0,1], \mathbb{R}) \rightarrow L([0,1], \mathbb{R})$ given by the formula

$$
C([0,1], \mathbb{R}) \ni u \longmapsto \ell u:=u(1) .
$$

We set $K:=[0,+\infty)$; the relation " $\geqslant_{K}$ " then coincides with the usual symbol " $\geq$ " and, hence, (4.11) always implies (4.10) in this case.

According to Example 3.12, operator (4.17) satisfies Assumption 3.11 whenever one can specify a nonnegative constant $B$ such that

$$
\int_{0}^{t} d s \leq B \sqrt{t}, \quad t \in[0,1]
$$

that is, if

$$
t \leq B \sqrt{t}, \quad t \in[0,1] .
$$


Relation (4.19) is satisfied, for example, with $B:=1$. Operator (4.17) is, obviously, $\phi$ regular. Indeed, with our definition of $\phi$, a function $u$ belongs to $C_{\phi}([0,1], \mathbb{R})$ if and only if

$$
|u(t)| \leq r \sqrt{t} \quad \forall t \in[0,1]
$$

for some $r>0$, and the number $\|u\|_{\phi}$ is equal to the greatest lower bound of all such $r$. At $t=0$, (4.20) yields $|u(1)| \leq\|u\|_{\phi}$, and, by virtue of (4.17), relation (3.5) is satisfied with $\omega(t)=1$ for a.e. $t \in[0,1]$.

We put

$$
y(t):=\epsilon, \quad t \in[0,1]
$$

where $\epsilon>0$. In view of (4.17) and (4.21), we have

$$
y(t)-\int_{0}^{t}(\ell y)(s) d s=\epsilon-\epsilon t=\epsilon(1-t) \geq 0, \quad t \in[0,1] .
$$

This means that inequality (4.13) holds for function (4.21) with $\tau=0$ and $\alpha=1$. Since $\epsilon$ is positive, the function $y$ also satisfies the corresponding condition (4.12).

Note now that condition (4.4) in our case has the form

$$
\sup _{t \in[0,1]} \frac{1}{\sqrt{t}}\left|\int_{0}^{t} f(s) d s+c t\right|<+\infty .
$$

For $c:=1$, relation (4.23) means the existence of a constant $\gamma$ such that

$$
\left|\int_{0}^{t} f(s) d s+t\right| \leq \gamma \sqrt{t}, \quad t \in[0,1]
$$

and is satisfied, for example, with $\gamma=2$ and

$$
f(t)=1, \quad t \in[0,1] .
$$

Thus, function (4.21) satisfies all the assumptions of Corollary 4.7, except the strict condition (4.13), instead of which the inequality

$$
y(t) \geq \int_{0}^{t}(\ell y)(s) d s, \quad t \in[0,1],
$$

is true. However, problem (4.15), (4.16) with $f$ given by (4.25) has no solutions.

Indeed, let $u$ be a solution of problem (4.15), (4.16) with $f \equiv 1$. Condition (4.16) implies, in particular, that

$$
u(0)=1
$$

Integrating (4.15) and taking (4.25) and (4.27) into account, we easily get that $u$ admits the representation

$$
u(t)=1+t(1+u(1)), \quad t \in[0,1]
$$


However, at the point $t=1$, equality (4.28) takes the form

$$
u(1)=2+u(1)
$$

and, hence, is not satisfied. This means that the initial value problem (4.15), (4.27) is unsolvable and, consequently, problem (4.15), (4.16) has no solutions. (Note that the function $u(t)=\lambda t, t \in[0,1]$, where $\lambda$ is an arbitrary real constant, is a solution of the homogeneous Cauchy problem $u^{\prime}=u(1), u(0)=0$.)

We have thus shown that, in the weakened form with $\alpha=1$, assumption (4.13) may not guarantee the solvability of problem (1.1), (1.2) for arbitrary $f$ and $c$ satisfying (4.4), and, hence, is strict in this sense. Clearly, the same is true for condition (4.3) appearing in Theorems 4.1 and 4.5.

Remark 4.9. The conditions in the theorems established above resemble those introduced in [6] for finite systems of linear functional differential equations determined by operators satisfying certain positivity conditions. It should be noted, however, that both the methods and results of [6] and of this paper are essentially different from one another.

Remark 4.10. Similarly to $[2,6]$, the theorems of the present paper allow one to obtain efficient conditions for the solvability of problem (1.1), (1.2) for various kinds of (in this case, generally speaking, infinite-dimensional) functional differential equations (1.1). Here, we restrict our consideration to the general theorems only.

\section{Auxiliary statements and proofs}

The proofs of the results formulated above use a number of auxiliary statements given in Sections 5.1, 5.2, and 5.3.

5.1. The space $C_{\phi}([a, b], X)$. Given a nonnegative continuous function $\phi:[a, b] \rightarrow \mathbb{R}$ such that

$$
\phi \not \equiv 0, \quad \phi^{-1}(0) \neq \varnothing,
$$

we set

$$
\left(T_{\phi} u\right)(t):=\frac{u(t)}{\phi(t)}, \quad t \in[a, b]
$$

for an arbitrary $u$ from $C_{\phi}([a, b], X)$. Note that, due to condition (2.3) imposed on $u$, the term on the right-hand side of (5.2) is well defined.

It is easy to see that formula (5.2) defines a linear operator from $C_{\phi}([a, b], X)$ to $B([a, b], X)$. This operator is bounded because, for $u \in C_{\phi}([a, b], X)$ (i.e., if $\|u\|_{\phi}<+\infty$, see $(2.4))$,

$$
\sup _{t \in[a, b]}\left\|\left(T_{\phi} u\right)(t)\right\|=\sup _{t \in[a, b]}\left\|\frac{u(t)}{\phi(t)}\right\|=\sup _{t \in[a, b]} \frac{\|u(t)\|}{\phi(t)}=\|u\|_{\phi}
$$

and, hence, $\left\|T_{\phi}\right\| \leq 1$. Moreover, mapping (5.2) has the following important property. 
Lemma 5.1. For an arbitrary continuous function $\phi:[a, b] \rightarrow[0,+\infty)$ satisfying (5.1), the set $T_{\phi} C_{\phi}([a, b], X)$ is a closed subset of $B([a, b], X)$ with respect to the norm (2.4).

Proof. Let $\left\{u_{m} \mid m \in \mathbb{N}\right\} \subset C_{\phi}([a, b], X)$ be a sequence such that

$$
\lim _{m \rightarrow+\infty} \sup _{t \in[a, b]}\left\|T_{\phi} u_{m}(t)-v^{*}(t)\right\|=0
$$

for a certain function $v^{*}$ from $B([a, b], X)$. We need to show that $v^{*}$ belongs to the image of $C_{\phi}([a, b], X)$ under the mapping $T_{\phi}$, that is, is representable as

$$
v^{*}(t)=\frac{u^{*}(t)}{\phi(t)}, \quad t \in[a, b]
$$

where $u^{*}:[a, b] \rightarrow X$ is a certain continuous function such that $\left\|u^{*}\right\|_{\phi}<+\infty$.

We put

$$
v_{m}(t):=\frac{u_{m}(t)}{\phi(t)}, \quad t \in[a, b], m=1,2, \ldots
$$

fix an arbitrary positive $\epsilon$, take some arbitrary point $t \in[a, b]$, and consider the difference $v^{*}(t+\delta) \phi(t+\delta)-v^{*}(t) \phi(t)$, where $\delta \in[a-t, b-t]$. We have

$$
\begin{aligned}
v^{*}(t+ & \delta) \phi(t+\delta)-v^{*}(t) \phi(t) \\
= & v^{*}(t+\delta) \phi(t+\delta)-v_{m}(t+\delta) \phi(t+\delta) \\
& +v_{m}(t+\delta) \phi(t+\delta)-v_{m}(t) \phi(t) \\
& +v_{m}(t) \phi(t)-v^{*}(t) \phi(t),
\end{aligned}
$$

whence

$$
\begin{aligned}
\| v^{*}( & t+\delta) \phi(t+\delta)-v^{*}(t) \phi(t) \| \\
\leq & \left\|v^{*}(t+\delta)-v_{m}(t+\delta)\right\| \phi(t+\delta) \\
& +\left\|v_{m}(t+\delta) \phi(t+\delta)-v_{m}(t) \phi(t)\right\| \\
& +\left\|v_{m}(t)-v^{*}(t)\right\| \phi(t) .
\end{aligned}
$$

We estimate the right-hand side of (5.8) from above.

Relation (5.4) means nothing but the convergence of the sequence $\left\{v_{m} \mid m \in \mathbb{N}\right\}$ to the function $v^{*}$ with respect to the norm (2.2). Therefore, for the given $\epsilon$, one can always specify a number $m_{\epsilon} \in \mathbb{N}$ such that

$$
\left\|v^{*}(t)-v_{m}(t)\right\| \phi(t)<\frac{\epsilon}{3 \max _{\xi \in[a, b]} \phi(\xi)}
$$

for all $t \in[a, b]$ and $m \geq m_{\epsilon}$. Recall that $\phi$ is not identically zero and, therefore, the righthand side term in (5.9) makes sense. 
According to (5.6), the function $[a, b] \ni t \mapsto v_{m}(t) \phi(t), m=1,2, \ldots$, coincides with the function $u_{m}$ belonging to $C_{\phi}([a, b], X)$. By the continuity of $u_{m}$, it follows that, for $m=m_{\epsilon}$,

$$
\left\|v_{m}(t+\delta) \phi(t+\delta)-v_{m}(t) \phi(t)\right\|<\frac{\epsilon}{3}
$$

whenever $|\delta|$ becomes less than a certain positive $\delta_{\epsilon}$. Estimating now the first and the third summand on the right-hand side of (5.8) by using (5.9) and taking (5.10) into account for the second one, we obtain that

$$
\left\|v^{*}(t+\delta) \phi(t+\delta)-v^{*}(t) \phi(t)\right\|<2 \frac{\epsilon}{3 \max _{\xi \in[a, b]} \phi(\xi)} \max _{s \in[a, b]} \phi(s)+\frac{\epsilon}{3}=\epsilon
$$

for arbitrary $t \in[a, b]$ and $\delta \in[a-t, b-t]$ satisfying the inequality $|\delta|<\delta_{\epsilon}$. By the arbitrariness of $\epsilon$, this means the continuity of the function $[a, b] \ni t \mapsto v^{*}(t) \phi(t)$ and, therefore, the function $v^{*}$ admits representation (5.5) with $u^{*}:=v^{*} \phi, y^{*} \in C_{\phi}([a, b], X)$.

Thus, the fulfilment of (5.4) for a sequence $\left\{u_{m} \mid m \in \mathbb{N}\right\} \subset C_{\phi}([a, b], X)$ and a function $v^{*}$ from $B([a, b], X)$ always implies the inclusion

$$
v^{*} \in T_{\phi} C_{\phi}([a, b], X),
$$

that is, the assertion of the lemma is true.

Lemma 5.2. $\left\langle C_{\phi}([a, b], X),\|\cdot\|_{\phi}\right\rangle$ is a Banach space.

Proof. It is clear from (2.4) that the mapping $C_{\phi}([a, b], X) \ni u \mapsto\|u\|_{\phi}$ satisfies the conditions $\|\alpha u\|_{\phi}=|\alpha|\|u\|_{\phi}$ and

$$
\left\|u_{1}+u_{2}\right\|_{\phi} \leq\left\|u_{1}\right\|_{\phi}+\left\|u_{2}\right\|_{\phi}
$$

for arbitrary $\left\{u, u_{1}, u_{2}\right\} \subset C_{\phi}([a, b], X)$ and $\alpha \in \mathbb{R}$. Furthermore, the relation $\|u\|_{\phi}=\eta$ yields the estimate $\|u(t)\| \leq \eta \phi(t)$ for all $t$ from $[a, b]$. Therefore, the equality $\|u\|_{\phi}=0$ implies that $u(t)=0$ for every $t \in[a, b]$.

It remains to prove the completeness of $C_{\phi}([a, b], X)$ with respect to the norm $\|\cdot\|_{\phi}$.

Let $\left\{u_{m} \mid m \in \mathbb{N}\right\}$ be a Cauchy sequence with respect to $\|\cdot\|_{\phi}$. This, in view of (2.4), means that, for an arbitrary positive $\epsilon$, there exists an $N \in \mathbb{N}$ such that

$$
\left\|u_{m+r}(t)-u_{m}(t)\right\| \leq \epsilon \phi(t), \quad t \in[a, b],
$$

for all $m \geq N$ and $r \geq 1$. In other words, the sequence

$$
v_{m}:=\frac{u_{m}}{\phi}, \quad m=1,2, \ldots
$$

is a Cauchy sequence in the space $B([a, b], X)$. The space $B([a, b], X)$ is complete with respect to the norm (2.2) and, therefore, there exists a bounded function $v^{*}:[a, b] \rightarrow X$ such that

$$
\lim _{m \rightarrow+\infty} \sup _{t \in[a, b]}\left\|v_{m}(t)-v^{*}(t)\right\|=0
$$


By virtue of Lemma 5.1, the function $v^{*}$ can be represented as

$$
v^{*}(t)=\frac{u^{*}(t)}{\phi(t)}, \quad t \in[a, b]
$$

where $u^{*} \in C_{\phi}([a, b], X)$. Hence, (5.16) can be rewritten as

$$
\lim _{m \rightarrow+\infty} \sup _{t \in[a, b]} \frac{\left\|u_{m}(t)-u^{*}(t)\right\|}{\phi(t)}=0
$$

or, which is the same, as

$$
\lim _{m \rightarrow+\infty}\left\|u_{m}-u^{*}\right\|_{\phi}=0
$$

Thus, an arbitrary Cauchy sequence converges in the space $C_{\phi}([a, b], X)$, and our lemma is proved.

5.2. Spectrum of linear operators vanishing on the blade of a wedge. Let $E$ be a Banach space and $K$ a wedge in it. The following statement is an extension of a well-known result (see, e.g., $[3,4]$ ) that establishes an upper bound for the spectrum of a linear operator preserving a solid wedge and dates back to a statement due to Kreĭn [5].

Theorem $5.3[7,9]$. Let $W$ be a solid wedge in $E$ and let $A: E \rightarrow E$ be a completely continuous linear operator such that $A(W) \subset W$ and

$$
W \cap(-W) \subset \operatorname{ker} A
$$

Then the existence of constants $\alpha \in[0,+\infty), m \in \mathbb{N}$, and an interior element $g$ in $W$ such that

$$
\alpha g-A^{m} g \in W
$$

implies the estimate

$$
r(A) \leq \sqrt[m]{\alpha}
$$

Here, as usual, $A(H):=\{A x \mid x \in H\}$ for all $H \subset X$.

5.3. The set $C_{K, \Omega, \phi}([a, b], X)$. Throughout this section, we fix a proper wedge $K$ in the Banach space $X$ and a set $\Omega \subset[a, b]$ such that the corresponding set $[a, b] \backslash \Omega$ is closed. We also choose a nonnegative continuous function $\phi:[a, b] \rightarrow \mathbb{R}$ satisfying conditions (5.1).

Let $C_{K, \Omega, \phi}([a, b], X)$ be the set of all continuous abstract functions $u:[a, b] \rightarrow X$ satisfying conditions (3.4) and (2.3):

$$
C_{K, \Omega, \phi}([a, b], X):=\left\{u \in C([a, b], X) \mid u(t) \geqslant_{K} 0 \forall t \in[a, b] \backslash \Omega \text {, and (2.3) holds }\right\} .
$$


Lemma 5.4. For an arbitrary proper wedge $K \subset X$, the set $C_{K, \Omega, \phi}([a, b], X)$ is a proper wedge in $C_{\phi}([a, b], X)$.

The structure of the blade of the wedge $C_{K, \Omega, \phi}([a, b], X)$ is described easily in terms of the blade of the wedge $K$.

Lemma 5.5. For an arbitrary wedge $K$ in $X$, the following equality is true:

$$
\text { blade } C_{K, \Omega, \phi}([a, b], X)=\left\{u \in C_{\phi}([a, b], X) \mid u([a, b] \backslash \Omega) \subset \text { blade } K\right\} .
$$

Lemmas 5.4 and 5.5 are proved similarly to the corresponding statements from [8].

Lemma 5.6. The wedge $C_{K, \Omega, \phi}([a, b], X)$ is solid whenever $K$ possesses the property indicated. In the case where the wedge $K$ is solid, a function $y$ belongs to the interior of $C_{K, \Omega, \phi}([a, b], X)$ if and only if relation (4.1) holds.

Proof. A function $y$ from $C_{\phi}([a, b], X)$ is an interior element of $C_{K, \Omega, \phi}([a, b], X)$ if and only if there exists a $\delta \in(0,+\infty)$ such that every function $u \in C_{\phi}([a, b], X)$ having the property

$$
\sup _{t \in[a, b]} \frac{1}{\phi(t)}\|u(t)-y(t)\|<\delta
$$

satisfies the inclusion $u([a, b] \backslash \Omega) \subset K$. This implies, in particular, that $y(t)$ is an interior point of $K$ for every $t$ from $[a, b] \backslash \Omega$, which means that condition (4.1) holds.

Assume now that the set $\{y(t) \mid t \in[a, b] \backslash \Omega\}$ is contained in the interior of the wedge $K$. Then, for every $t \in[a, b] \backslash \Omega$, one can specify a positive number $\delta$ such that all elements $x \in X$ satisfying the relation

$$
\|y(t)-x\|<\delta \phi(t)
$$

belong to $K$. Let $\delta(t)$ be the least upper bound of all such $\delta$ at the point $t$ from $[a, b] \backslash \Omega$. By taking the continuity of $y$ and $\phi$ into account, one can show that the function $\delta$ : $[a, b] \backslash \Omega \rightarrow(0,+\infty)$ is continuous.

We put $\delta_{*}:=\inf _{t \in[a, b] \backslash \Omega} \delta(t)$. The number $\delta_{*}$ is strictly positive. Indeed, in the contrary case, there exists a sequence $\left\{t_{k} \mid k \in \mathbb{N}\right\} \subset[a, b] \backslash \Omega$ such that $\lim _{k \rightarrow+\infty} \delta\left(t_{k}\right)=0$. The compactness of the interval $[a, b]$ implies the existence of a sequence $\left\{k_{n} \mid n \in \mathbb{N}\right\} \subset$ $\mathbb{N}$ and a point $t_{*}$ such that $\lim _{n \rightarrow+\infty} t_{k_{n}}=t_{*}$. The point $t_{*}$ belongs to $[a, b] \backslash \Omega$ because the set mentioned is closed. Hence, $\lim _{n \rightarrow+\infty} \delta\left(t_{k_{n}}\right)=0$ and, therefore, $\delta\left(t_{*}\right)=0$, contrary to the definition of $\delta(\cdot)$ and, hence, to the assumption that $y(t)$ is contained in the interior of $K$ for all $t$ from $[a, b] \backslash \Omega$.

Thus, we can put, for example, $\delta=(1 / 2) \delta_{*}$ in (5.25) at every point $t$ from $[a, b] \backslash \Omega$.

5.4. The operator $\mathscr{E}_{\tau, \ell}$. Given a linear operator $\ell: C([a, b], X) \rightarrow L([a, b], X)$, we introduce the mapping $\mathscr{F}_{\tau, \ell}$ by putting

$$
\left(\mathscr{F}_{\tau, \ell} u\right)(t):=\int_{\tau}^{t}(\ell u)(s) d s, \quad t \in[a, b] .
$$


It is clear that $\mathscr{F}_{\tau, \ell}$ is a linear operator transforming the space $C([a, b], X)$ into itself. Moreover, the following statement is true.

Lemma 5.7. For an arbitrary linear operator $\ell: C([a, b], X) \rightarrow L([a, b], X)$ satisfying Assumption 3.11, the corresponding linear mapping $\mathscr{F}_{\tau, \ell}: C([a, b], X) \rightarrow C([a, b], X)$ leaves invariant the set $C_{\phi}([a, b], X)$.

Here, as in Section 5.3, the set $[a, b] \backslash \Omega$ is closed and $\phi:[a, b] \rightarrow[0,+\infty)$ is a certain continuous function not identically equal to zero on $[0,1]$.

Proof. Indeed, we take an arbitrary function $u:[a, b] \rightarrow X$ belonging to $C_{\phi}([a, b], X)$, that is, a continuous function satisfying condition (2.3). By virtue of Assumption 3.11, there exists some nonnegative constant $\beta_{u}$ such that inequality (3.7) holds, which means that the function

$$
[a, b] \ni t \longmapsto \int_{\tau}^{t}(\ell u)(\xi) d \xi
$$

is an element of $C_{\phi}([a, b], X)$.

Lemma 5.8. If the operator $\ell: C([a, b], X) \rightarrow L([a, b], X)$ is $(\tau, \Omega, \phi)$-positive with respect to the wedge $K$ in $X$, then the corresponding operator (5.27) leaves invariant the wedge $C_{K, \Omega, \phi}([a, b], X)$ :

$$
\mathscr{J}_{\tau, \ell} C_{K, \Omega, \phi}([a, b], X) \subset C_{K, \Omega, \phi}([a, b], X) .
$$

We also need the following properties of operator (5.27).

Lemma 5.9. For every linear operator $\ell: C([a, b], X) \rightarrow L([a, b], X)$ belonging to the set $\mathscr{B}_{K, \phi}(\tau, \Omega ;[a, b], X)$, the corresponding mapping $\mathscr{I}_{\tau, \ell}$ vanishes on the blade of the wedge $C_{K, \Omega, \phi}([a, b], X)$.

Lemma 5.10. For every $\phi$-regular linear operator $\ell: C([a, b], X) \rightarrow L([a, b], X)$, the corresponding linear mapping $\mathscr{S}_{\tau, \ell}: C_{\phi}([a, b], X) \rightarrow C_{\phi}([a, b], X)$ is compact.

Lemmas 5.8, 5.9, and 5.10 are proved similarly to the corresponding statements from $[8]$.

5.5. Proof of Theorem 4.1. We first formulate the following obvious lemma concerning the inhomogeneous initial value problem (1.1), (4.7) associated with the original problem (1.1), (1.2).

Lemma 5.11. For an arbitrary $\tau \in[a, b]$, the set of solutions of the Cauchy problem (1.1), (4.7) coincides with the set of continuous solutions of the functional equation

$$
u=\mathscr{E}_{\tau, \ell} u+f_{\tau, c} .
$$

Remark 5.12. It is clear that every solution of (5.30) is absolutely continuous. 
Note that, when $\tau \in \phi^{-1}(0)$, the fulfilment of condition (1.2) for a function $u$ from $C([a, b], X)$ implies, in particular, that (4.7) holds. This means that, under the assumptions of Theorem 4.1, a solution of (1.1), (1.2) always satisfies the Cauchy problem (1.1), (4.7).

We show that problem (1.1), (4.7) is uniquely solvable for arbitrary $c \in X$ and $f \in$ $L([a, b], X)$ satisfying condition (4.4). In view of Lemma 5.11, it will suffice to prove the unique solvability of (5.30) for arbitrary $f$ and $c$ for which (4.4) is true. Recall that $f_{\tau, c}$ in (5.30) is the function constructed from $f$ and $c$ by formula (4.6).

We first consider the corresponding semihomogeneous problem, that is, the Cauchy problem (1.1), (4.7) with (4.7) replaced by the homogeneous condition

$$
u(\tau)=0
$$

By Lemma 5.11, problem (1.1), (5.31) is equivalent to the equation

$$
u=\mathscr{g}_{\tau, \ell} u+\int_{\tau}^{\cdot} f(\xi) d \xi
$$

According to Lemma 5.7, the mapping $\mathscr{f}_{\tau, \ell}$ leaves invariant the set $C_{\phi}([a, b], X)$. Therefore, in order to prove the unique solvability of (5.32) for all integrable $f$ satisfying (4.4) with $c=0$, that is, satisfying the condition

$$
\sup _{t \in[a, b]} \frac{1}{\phi(t)}\left\|\int_{\tau}^{t} f(s) d s\right\|<+\infty,
$$

it is sufficient to prove that the spectrum of $\mathscr{F}_{\tau, \ell}$ considered as an operator in the space $C_{\phi}([a, b], X)$ is contained inside the unit circle with the centre at zero.

We will show that, under the conditions assumed, the spectral radius $\varrho_{\ell}$ of the operator $\mathscr{F}_{\tau, \ell}: C_{\phi}([a, b], X) \rightarrow C_{\phi}([a, b], X)$ admits the estimate

$$
\varrho_{\ell} \leq \alpha
$$

Indeed, condition (4.3) assumed in Theorem 4.1 for a continuous function $y:[a, b] \rightarrow X$ can be rewritten as

$$
\alpha y(t) \geqslant_{K}\left(\mathscr{F}_{\tau, \ell} y\right)(t) \quad \forall t \in[a, b] \backslash \Omega
$$

which, in view of (4.2), means nothing but the inclusion

$$
\alpha y-\mathscr{F}_{\tau, \ell} y \in C_{K, \Omega, \phi}([a, b], X) .
$$

The set $C_{K, \Omega, \phi}([a, b], X)$, as we know from Lemma 5.4, forms a proper wedge in the space $C([a, b], X)$. Furthermore, in view of Lemma 5.6, the function $y$ satisfying condition (4.1) belongs to the interior of the wedge $C_{K, \Omega, \phi}([a, b], X)$.

Note also that, in view of Lemma 5.9, the inclusion $\ell \in \mathscr{B}_{K, \phi}(\tau, \Omega ;[a, b], X)$ implies that the mapping $\mathscr{S}_{\tau, \ell}: C([a, b], X) \rightarrow C([a, b], X)$ vanishes on the blade of the wedge $C_{K, \Omega, \phi}([a, b], X)$. According to Lemma 5.10, the $\phi$-regularity of $\ell$ implies the complete continuity of the corresponding linear mapping $\mathscr{F}_{\tau, \ell}$. Finally, in view of Lemma 5.8, 
the $(\tau, \Omega, \phi)$-positivity of $\ell$ with respect to $K$ implies that the operator $\mathscr{J}_{\tau, \ell}$ leaves invariant the wedge $C_{K, \Omega, \phi}([a, b], X)$.

Applying Theorem 5.3 with $m=1, E=C_{\phi}([a, b], X), W=C_{K, \Omega, \phi}([a, b], X), A=\mathscr{J}_{\tau, \ell}$, and $g=y$, we establish inequality (5.34). In view of the condition $0 \leq \alpha<1$, this, as is well known, guarantees the convergence of the series

$$
u:=f_{\tau, 0}+\mathscr{E}_{\tau, \ell} f_{\tau, 0}+\mathscr{g}_{\tau, \ell}^{2} f_{\tau, 0}+\cdots
$$

to the unique solution $u$ of (5.32) or, which is the same, of the semihomogeneous Cauchy problem (1.1), (5.31). Here, the function $f_{\tau, 0}$ is given by formula (4.6) with $c=0$.

Now let $u$ be a solution of the inhomogeneous Cauchy problem (1.1), (4.7). Putting $\tilde{u}:=u-c$ and $\tilde{f}:=f+\ell c$, we find that the function $\tilde{u}$ satisfies the semihomogeneous problem (1.1), (5.31) with $f$ replaced by $\tilde{f}$. Thus, it is easy to show that, under the conditions assumed, the unique solution of the inhomogeneous initial value problem (1.1), (4.7) is given by the series

$$
u:=f_{\tau, c}+\mathscr{F}_{\tau, \ell} f_{\tau, c}+\mathscr{E}_{\tau, \ell}^{2} f_{\tau, c}+\cdots .
$$

Clearly, series (5.38) coincides with (4.5).

Property (3.4) of the solution of problem (1.1), (1.2) follows immediately from its series representation (5.38) and inclusion (5.29) because $C_{K, \Omega, \phi}([a, b], X)$ is a closed set possessing property (1) of Definition 3.1.

5.6. Proof of Theorem 4.5. In the case where $K$ is a cone, according to formula (5.24), the equality

$$
\text { blade } C_{K, \Omega, \phi}([a, b], X)=\left\{u \in C_{\phi}([a, b], X) \mid u \text { vanishes outside } \Omega\right\}
$$

is true. Therefore, Theorem 4.5 is a consequence of Theorem 4.1.

5.7. Proof of Corollary 4.7. It is sufficient to apply Theorem 4.5 with $\Omega=\varnothing$.

\section{Acknowledgment}

The research of the second author was supported in part by the NATO Science Fellowships Programme for the Czech Republic, Grant 7/2003.

\section{References}

[1] I. A. Bakhtin, M. A. Krasnosel'skii, and V. Y. Stetsenko, On the continuity of linear positive operators, Sibirsk. Mat. Zh. 3 (1962), no. 1, 156-160.

[2] N. Z. Dilnaya and A. Rontó, Some new solvability conditions of the Cauchy problem for systems of linear functional differential equations, Ukrain. Math. J. 56 (2004), no. 7, 867-884.

[3] M. A. Krasnosel'skii, J. A. Lifshits, and A. V. Sobolev, Positive Linear Systems. The Method of Positive Operators, Sigma Series in Applied Mathematics, vol. 5, Heldermann Verlag, Berlin, 1989.

[4] M. A. Krasnosel'skii, G. M. Vaĭnikko, P. P. Zabreı̆ko, Y. B. Rutitskii, and V. Y. Stetsenko, Approximate Solution of Operator Equations, Wolters-Noordhoff Publishing, Groningen, 1972. 
[5] M. G. Krein and M. A. Rutman, Linear Operators Leaving Invariant a Cone in a Banach Space, no. 26, Amer. Math. Soc. Transl., American Mathematical Society, Rhode Island, 1950.

[6] A. Rontó, Exact solvability conditions of the Cauchy problem for systems of linear first-order functional differential equations determined by $\left(\sigma_{1}, \sigma_{2}, \ldots, \sigma_{n} ; \tau\right)$-positive operators, Ukrainian Math. J. 55 (2003), no. 11, 1853-1884.

[7] _ On substantial eigenvalues of linear operators leaving invariant a closed wedge, Colloquium on Differential and Difference Equations, CDDE 2002 (Brno), Folia Fac. Sci. Natur. Univ. Masaryk. Brun. Math., vol. 13, Masaryk Univ., Brno, 2003, pp. 235-246.

[8] A. Rontó and J. Šremr, Abstract differential inequalities and the Cauchy problem for infinitedimensional linear functional differential equations, to appear in Archives Inequalities Appl.

[9] A. Rontó, Bounds for the spectrum of compact linear operators in a preordered Banach space, to appear in Abstr. Appl. Anal.

Ravi P. Agarwal: Department of Mathematical Sciences, Florida Institute of Technology, 150 West University Boulevard, Melbourne, FL 32901-6975, USA

E-mail address: agarwal@fit.edu

Andrei Rontó: Institute of Mathematics, National Academy of Sciences of Ukraine, 3 Tereschenkovskaya Street, 01601 Kiev, Ukraine

Current address: Mathematical Institute, Academy of Sciences of the Czech Republic, Žižkova 22, 61662 Brno, Czech Republic

E-mail address: ronto@ipm.cz 